\title{
Tunneling Effect Enhanced by Pd Screening as Main LENR Mechanism: A Brief Theoretical Impression
}

\author{
Frisone Fulvio \\ Department of Physics, University of Catania, Via Santa Sofia 64, I-95123 Catania, Italy
}

\begin{abstract}
In this paper the main features of tunneling travelling are illustrated between two deuterons within a lattice. Considering the screening effect due to lattice electrons we compared the d-d fusion rate evaluated from different authors assuming different screening efficiency and different $d-d$ potentials. Then, we proposed an effective potential which describes very well the attractive contribution due to plasmon exchange between two deuterons and by means of it we will compute the d-d fusion rates for different energy values.
\end{abstract}

Key words: Dissociation energy, interaction between two deuterons, effect tunnel.

\section{Introduction}

The first cold fusion phenomenon has been observed in the 1926 by Paneth and Peters that passed $\mathrm{H}_{2}$ through a high temperature $\mathrm{Pd}$ capillary tube and they observed He spectral line [1]. Of course this episode does not sign the rise of a new branch of science since they, subsequently, declared that the helium was released from the heated glass container. Then, for about 60 years, nobody spoke about low energy nuclear reaction, until, on March 23, 1989, Fleischmann and Pons declared the achievement of a deuterons fusion at room temperature in a $\mathrm{Pd}$ electrolytic cell using $\mathrm{D}_{2} \mathrm{O}$ [2].

Since their discovery, a large amount of efforts have been spent in order to reproduce the fusion but actually the experimental knowledge and the know-how is not so far robust. However, the evidence of anomalous nuclear phenomenon had been built up in these last years [3-6].

In this paper, we want to summarize some theoretical efforts in order to understand the probable mechanism of cold fusion. In particular we focus on

Corresponding author: Frisone Fulvio, nuclear researcher, research fields: theoretical physics, nuclear physics, low energy nuclear reactions, structure of matter, condensed matter science of metals. tunneling travelling of Coulomb barrier existing between two deuterons. More exactly, it will analyze the possible contributions of the lattice in order to enhance the tunneling probability and, in this way, we will find that there is a real mechanism by means of which this probability is increased a lot: this mechanism is the screening effect due to the d-shell electrons of palladium lattice.

\section{Tunneling in Molecular $\mathbf{D}_{2}$}

After the nuclear disintegration had been discovered, it soon became clear that the laws of classical dynamics were not able to reproduce some experimental regularities as the radioactive decay law. This law involves that the time of disintegration of an atom is independent of the previous history and of its physical condition, in other words the behaviour of particles is governed by probability. Gamow [7] demonstrated that using the Copenhagen interpretation of Schrödinger equation it was possible to obtain the correct decay law expression. In this way the tunnel effect was universally accepted. Actually we are not to able to understand transmutation and disintegration phenomena without the non-classical travelling of potential barrier.

In this time, the tunneling effect is a very important statement of physics "behaviour" of microscopic 
particles; for example many of the microelectronic devices as flash memories [8] base themselves on Fowler-Nordheim tunneling.

Now, the fusion process established in terms of penetration of a particle of energy $E$ in a region classically forbidden whose potential is $V$, the fusion reaction rate $\Lambda\left(\mathrm{sec}^{-1}\right)$ will be determined, according to quantum mechanics, from the following expression:

$$
\Lambda=A \mid \psi\left(r_{o}\right)^{2}
$$

Here $\mathrm{A}$ is the nuclear reaction constant obtained from measured cross sections, the probability $\left|\psi\left(r_{0}\right)\right|^{2}$ is the square modulus of the inter-particles wave-function, and $r_{0}$ is the point of forbidden zone. Finally it is demonstrated that for a Coulomb potential:

$$
\begin{gathered}
\left|\psi\left(r_{0}\right)\right|^{2}=\left|\frac{k\left(r_{e}\right)}{k\left(r_{0}\right)}\right| \exp \left\{-\frac{2}{\hbar} \int_{r_{0}}^{r^{\prime}} \sqrt{2 \mu(V-E))} d r\right\} \\
k(r)=\frac{\sqrt{2 \mu(V(r)-E))}}{\hbar}
\end{gathered}
$$

and $r$ ' is the classical turning point.

Here $\mu$ is the mass of particle incoming, $r_{0}$ is a point within the forbidden region, $E$ is the energy of particle, and $k\left(r_{e}\right)$ is the wave number of the zero point oscillation:

$$
\frac{\hbar^{2} k^{2}\left(r_{e}\right)}{2 \mu}=E
$$

Of course, the pre-factor of exponential is about 1 and the exponential term is known as Gamow-amplitude. More exactly we define as Gamow-factor:

$$
\eta_{G}\left(r_{0}\right)=\exp \left\{-\frac{1}{\hbar} \int_{r}^{r_{0}} \sqrt{2 \mu(V-E))} d r\right\}
$$

Now compute the fusion probability between two nuclei of molecular $D_{2}$. In this case we must consider that the average distance between two deuterons, within the $D_{2}$-molecule, is $r_{0} \approx 0.74 \stackrel{\circ}{A}$ while the distance at which the nuclear force takes place is $r \approx$ 20 F. Then if we put $V=\alpha / r$, i.e. the Coulomb potential, and with $\mu$ label the reduced mass, we will compute $k \approx 1$. Finally for $E \approx 0$ we can evaluate:

$$
\eta_{G}(r)=\exp -\left\{\sqrt{8 \alpha \mu}\left(r_{0}^{1 / 2}-r^{1 / 2}\right)\right\} \approx 10^{-60}
$$

Using $A=10^{22} \sec ^{-1}$, we obtain $\Lambda=10^{-23} \sec ^{-1}$. It means that no detectable process can take place!

\section{Deuterons Tunneling as Probable Cold Fusion Mechanism}

In this case the $\mathrm{d}$-d fusion processes involved are:

$$
\begin{gathered}
d+d \rightarrow{ }^{3} \mathrm{He}+n \\
d+d \rightarrow t+p \\
d+d \rightarrow{ }^{4} \mathrm{He}+\gamma
\end{gathered}
$$

In the previous section, we have computed that the fusion probability between two deuterons within molecular $\mathrm{D}_{2}$ is very low. But at one time evaluated in the 1986, this fusion rate can be reduced [9]. In fact some mechanisms, as the replacing of the electron in a molecular hydrogen ion with a negatively charged muon, are able to increase the reduced mass and then to decrease the Gamow-factor [10]. More exactly, Siclen and Jones, starting from the possibility of creating pressures of several million atmospheres presented by diamond-anvil cell, have considered deuterons fusion rates as function of pressure and, then, of the inter-nuclear distance obtaining an theoretical average fusion rate of $10^{-74} \mathrm{sec}^{-1}$. They discussed in this way: in a molecular hydrogen the potential has the trend shown in Fig. 1. In this case, a Morse potential is used in the interval that includes the inner turning point $r_{a}$ and continues on toward $r=0$, near which it is connected with the repulsive Coulomb potential $1 / \mathrm{r}$. The expression is:

$$
V(r)=D e\left[e^{-2 \gamma\left(r-r_{0}\right)}-2 e^{-\gamma\left(r-r_{0}\right)}\right]
$$




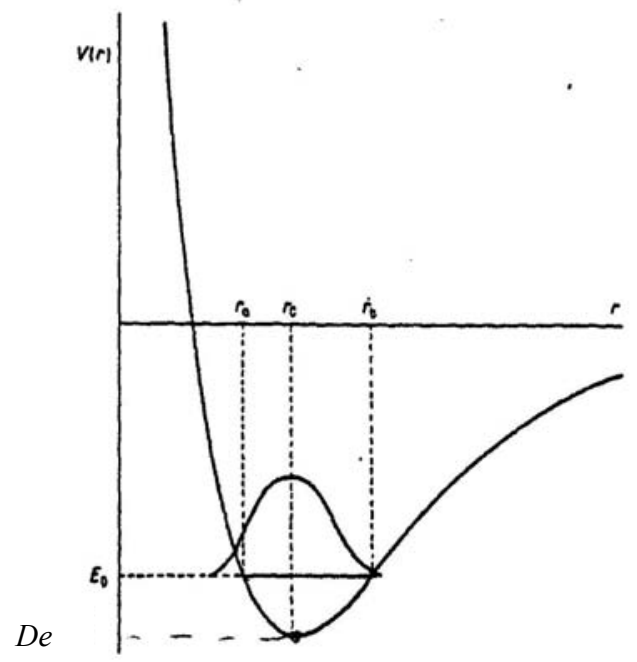

Fig. 1 Molecular potential energy curve and ground-state vibrational wave-function for the relative motion of the two nuclei. The points $r_{a}, r_{b}$ and $r_{0}$ are the classical turning points and the equilibrium inter-nuclear separation, respectively [9].

here $D e$ is the depth of the potential well that is roughly equal to the dissociation energy and $\gamma$ is related to the anharmonicity constant which is a measure of the curvature of the Morse potential well.

Since the vibrational levels of Morse potential can be written in this way [11]:

$$
E_{v}=-D e\left[1-\frac{\gamma \hbar}{\sqrt{2 \mu D} e}\left(v+\frac{1}{2}\right)\right]^{2}
$$

it is possible to evaluate by fitting the constant $\gamma$ and $D e$ and $r_{0}$. The authors [9] computed (in units $e^{2} / a_{0}$ and $a_{0}=$ Bohr radius):

$$
\begin{gathered}
D e=0.1743 \\
\gamma=1.04 \\
r_{0}=1.4
\end{gathered}
$$

The molecular wave-function has been evaluated following the method proposed by Langer in Ref. [12] which now we will briefly illustrate. The radial part of Schrödinger equation is:

$$
\begin{gathered}
{\left[\frac{d^{2}}{d r^{2}}+Q_{0}^{2}(r)\right] \chi(r)=0} \\
Q_{0}^{2}(r)=\frac{2 \mu}{\hbar^{2}}[E-V(r)]-\frac{J(J+1)}{r^{2}}
\end{gathered}
$$

$$
\chi(r)=r \psi(r) .
$$

The ground $v=J=0$ molecular wave-function in the interaction region is thus found to be:

$$
\begin{gathered}
\psi(r)=\frac{1}{4}\left(\frac{\alpha^{2}}{\pi^{3}}\right)^{1 / 4} \eta \\
\eta=\exp \left\{-\frac{1}{2}\left[\int_{r}^{r_{0}}\left(2|Q(r)|-\frac{1}{r}\right) d r+\ln \frac{r_{a}}{2}\right]\right\} \\
\alpha=\frac{\mu \omega}{\hbar} \\
Q^{2}(r)=\frac{2 \mu}{\hbar^{2}}[E-V(r)]-\frac{(J+1 / 2)^{2}}{r^{2}}
\end{gathered}
$$

where $V(r)$ is the coulomb potential for $r<\rho$ and the Morse potential for $r>\rho$ being $\rho$ the point at which the Morse potential is connected to the purely Coulomb potential.

Using these results Van Siclen ad Jones demonstrated the possibility of scaling down of repulsive effect between two deuterons. Moreover they showed that the average fusion rate was much more sensitive to the choice of $\rho$ than $r$ (see Table 1).

\section{The Screening Effect}

In 1989, Fleischmann and Pons observed that the establishment of negative over-potential on the outgoing palladium interface shows that the chemical potential can be raised to high values and it means that within palladium it is possible [13] to have an astronomical pressure of about $10^{26} \mathrm{~atm}$. After the Fleischmann and Pons experiments had been published, it soon became clearly the main role of palladium lattice as catalyzing.

To illustrate this topic, we report the argument of Horowitz [14].

The electrons in a metal should become a Fermi gas and the hydrogen nuclei interacting via screened Coulomb potential. The effective potential between two nuclei $V(r)$ which includes the effects of electron screening is given, in a simple Thomas-Fermi model, by: 
Table 1 Fusion rates evaluated in Ref. [9] as function of $\rho$ (point where the Morse potential is linked by Coulomb), and $r$ (force nuclear radius). The distance is reported in units of the Bohr radius.

\begin{tabular}{lll}
\hline$R$ & $P$ & $\Delta\left(\mathrm{sec}^{-1}\right)$ \\
\hline 0 & 0.4 & $3.8 \times 10^{-70}$ \\
0 & 0.5 & $1.3 \times 10^{-69}$ \\
$10^{-3}$ & 0.5 & $1.3 \times 10^{-64}$ \\
$10^{-3}$ & 0.5 & $2.3 \times 10^{-60}$ \\
$10^{-3}$ & 0.5 & $6.8 \times 10^{-58}$ \\
0 & 0.5 & $5.8 \times 10^{-54}$ \\
\hline
\end{tabular}

$$
V(r)=\frac{e^{2}}{r} \exp \left[-\frac{r}{\lambda}\right]
$$

of course $\lambda$ is the screening length and depends on density. But for $\mathrm{r}<<\lambda$ we can write at first order:

$$
V(r) f=\frac{e^{2}}{r}-V_{0}
$$

This constant $V_{0}$ would be just the difference between electronic energy of an $\mathrm{He}$ isolated atom $(-79.0 \mathrm{eV})$ and the binding energy of two $\mathrm{H}$ atoms $(-51.8 \mathrm{eV})$. The fusion rate has been evaluated using:

$$
A=v P_{n}
$$

where $v$ is the vibrational frequency of the crystal's zero point motion ( $h v$ is about $1 \mathrm{eV}$ ) and $P_{\mathrm{n}}$ is the probability of a $\mathrm{d}$-d nuclear reaction once the nuclei have made it to $r_{n}$. In other words the fusion rate is calculated by multiplying $P$ by the frequency of attacks on the Coulomb barrier and the probability of a nuclear reaction.

Put $h v=1 \mathrm{eV}$ and $P_{n}=1$ (for $\mathrm{d}-\mathrm{d}$ reaction), Horowitz computes $\Lambda=10^{-70} \mathrm{sec}^{-1}$, for $r_{0}=0.5 \AA$, but $\Lambda=10^{-25} \mathrm{sec}^{-1}$ for $r_{0}=0.1 \AA$.

Similar argumentations were proposed by Giuliano Preparata but started from a new formulation of condensed matter theory known as Coherence Theory. In fact, according to the Coherence Theory of Condensed Matter we can visualize the plasma formed by $d$-shell electrons as consisting of charged shells of charge $n_{d} e$ (for Palladium $n_{d}=10$ ) radius $r_{d}=1 \AA$ and thickness a fraction of one $\AA$. The classical plasma frequency will be:

$$
\omega_{d}=\frac{e}{\sqrt{m}} \sqrt{\frac{n_{d} N}{V}}
$$

According to the coherence theory of matter we must adjust this plasma frequency of a factor 1.38 . We can understand this correction observing that the formula (22) is obtained assuming a uniform d-electron charge distribution. But of course the d-electron plasma is localized in a shell of radius $R$ (that is about $1 \AA$ ), so the geometrical contribution is:

$$
\sqrt{\frac{6}{\pi}}=1.38
$$

and finally we can compute:

$$
\omega_{d}=41.5 \mathrm{eV} / \hbar
$$

These charge oscillations produce a screening potential having an harmonic feature:

$$
e V(r)=-Z_{d} \frac{k e^{2}}{2 a_{0}} r^{2}
$$

In Ref. [15] putting $Z_{\mathrm{d}}=10 / 3$ and $a_{0}=0.7 \AA$, it is evaluated a screening potential of about $85 \mathrm{eV}$. It means that within a palladium lattice the Coulomb potential between two deuterons has the following expression:

$$
V(r)=\frac{e^{2}}{r}-85 e V
$$

In this case, it is very easy to evaluate the intermolecular distance between two deuterons which can reach the value of $0.165 \AA$. Finally by 
means of Eq. (1) and using $A=10^{22} \mathrm{sec}^{-1}$, it is computed $\Lambda=10^{-22} \mathrm{sec}^{-1}$. This last value of fusion rate has been experimentally checked [16, 17], and for this reason we believe that it is correct. We conclude this section affirming that within a lattice, according to the quantum mechanics principles, the fusion probability becomes theoretically possible and experimentally observable.

\section{Proposal of an Effective Potential}

From the results reported in the previous sections, it appears clear that within a lattice the $d$-d reactions take place in a $D_{2}$-molecule whose inter-nuclear distance is reduced by screening effects. More exactly by means of works reported in Refs. [9, 14, 15], we can say that within a lattice:

(1) A compound potential as which was used by Siclen and Jones is a likely molecular potential.

(2) A screening effect that is able to reduce the inter-nuclear distance takes place

For this reason, starting from the idea of Jones and Siclen, we tried to find a $d-d$ effective potential that distance smaller than $\rho$ (i.e. the point where the attractive molecular potential is linked with the repulsive core) gives about the Coulomb feature while, for the distance bigger than the Morse potential is obtained. In Ref. [18], to fit such a "Coulomb-Morse linked" potential we have proposed the following effective potential:

$$
\begin{gathered}
V(r)=k \frac{e^{2}}{r}\left(V_{M}(r)-\frac{A}{r}\right) \\
V_{M}(r)=D^{\prime}\left[e^{-2 \gamma\left(r-r_{0}^{\prime}\right)}-2 e^{-\gamma\left(r-r_{0}^{\prime}\right)}\right] .
\end{gathered}
$$

Here $A, D^{\prime}, \gamma$ and $r_{0}$ are parameters to be determined by means of fitting.

Of course, the problem is to know the physical values that characterize, from a point of numerical view, a potential like that is used by Siclen and Jones. More exactly the principal issue, that we must solve, is to estimate:
- the point where the Morse potential is linked by Coulomb curve (i.e. $\rho$ ),

- the equilibrium distance, and

- the well depth.

In order to evaluate the parameter relative to first bullet, we used the screening value proposed by Preparata (average: $85 \mathrm{eV}$ ) as starting point.

In this way remembering Eq. (26) we compute $\rho=$ $V_{0} / 26.9$ and at last $\rho=0.165 \AA$.

Regarding the other topics (i.e. equilibrium distance $r_{0}$ and disassociation energy $D e$ ) we have discussed in this way.

In the ionized molecular hydrogen the equilibrium distance is about $1.06 \AA$, but in the neutral molecular hydrogen it is about $0.7 \AA$. We can interpret the results saying that the screening potential due to second electron whose magnitude (in units $e^{2} / a_{0}$ and $a_{0}=$ Bohr radius) is:

$$
2 \cdot \frac{26,9}{a_{0}}=53.8 \mathrm{eV}
$$

(here $a_{0}$ is the Bohr radius), reduces of about $34 \%$ the equilibrium distance.

Therefore, a screening magnitude of $85 \mathrm{eV}$ will be able to reduce this distance of about $50 \%$. Applying this argumentation to the neutral molecular hydrogen (and/or also to $D_{2}$-molecule, see Ref. [19]) we can suppose that inside the lattice the equilibrium distance between two nuclei of a $D_{2}$-molecule is about 0.7 to $0.35 \AA$.

A similar argumentation has been reported in order to evaluate the dissociation energy. In fact, we know that the $\mathrm{H}_{2}{ }^{+}$dissociation energy is about $2.7 \mathrm{eV}$,

Table 2 The left column shows the physical quantities that must characterize the potential Eq. (27); the right the model parameter values that are to be used in the Eq. (27) in order to obtain the physical values reported in the left column.

\begin{tabular}{ll}
\hline$\rho=0.165 \AA$ & $A=0.0001$ \\
$r_{0}=0.35 \AA$ & $r_{0}^{\prime}=0.99$ \\
$D e=9.34 \mathrm{eV}$ & $D^{\prime}=0.28$ \\
& $\gamma=1.04$ \\
\hline
\end{tabular}




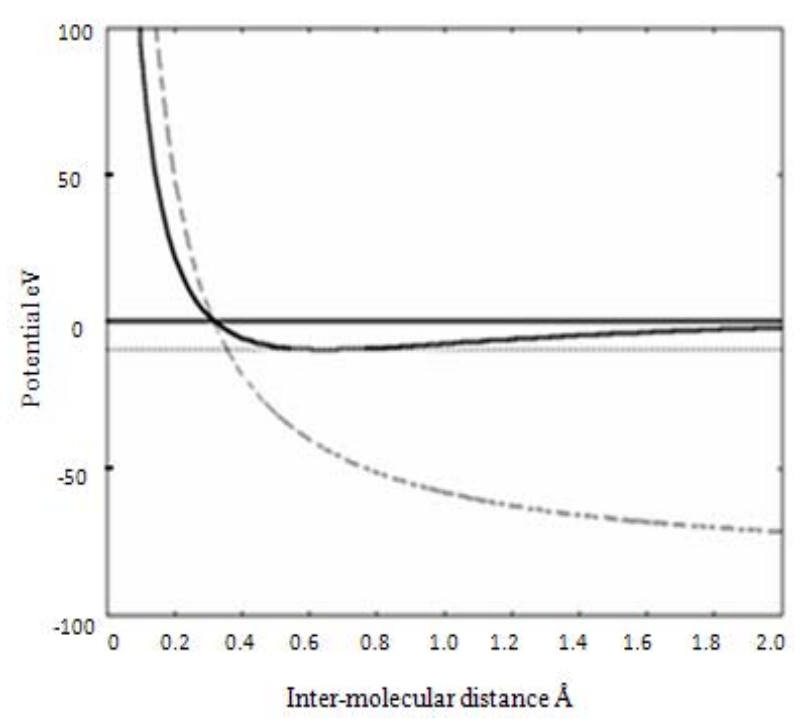

Fig. 2 The solid line shows the features of potential Eq. (27) computed using the values reported in Table 2.

whereas the $\mathrm{H}_{2}$ dissociation energy is about $4.6 \mathrm{eV}$, i.e. a scaling of equilibrium distance of $\pm 34 \%$ is able to produce a energy growth of $\pm 70 \%$. Then, we suppose that the scaling of $50 \%$, in the molecular $\mathrm{H}_{2}$ equilibrium distance, is able to increase the disassociation energy of about $\pm 103 \%$. In this way it has computed a dissociation energy of $9.34 \mathrm{eV}$.

In Table 2 , the $\rho, r_{0}$ and $D$ evaluations are reported and supposed and the parameters values of potential Eq. (27) are able to reproduce these quantities, while Fig. 2 shows the feature of potential Eq. (27) obtained using the values of Table 2 . Note the good agreement with the coulomb potential for $r<\rho$.

The dashed line is the Coulomb potential (26). Note that they cross the $x$-axes in the same point. In the $\mathrm{x}$-axis it is reported the distance in Bohr radius unit and on the $y$-axis the energy in $\mathrm{eV}$.

Of course, these argumentations are rough, but we believe that they are able to give a reasonable start point.

A better evaluation of point $\rho$, equilibrium distance and disassociation energy can be obtained from many-body theory. In fact as pointed out in Ref. [20], the deuteron interaction with the collectives plasmon excitations of the palladium produces a strong attractive potential. This attractive force is due to exchange of plasmons in Ref. [20] the authors consider only two plasmon excitations at $7.5 \mathrm{eV}$ and $26.5 \mathrm{eV}$ between two deuteron-lines as reported in Fig. 3.

Taking into account the role of coupling between deuteron and plasmons, in Ref. [20] the authors have numerically evaluated a $d-d$ potential having the features of potential Eq. (26). More exactly in their case $\rho$ is about $\pm 50 \mathrm{eV} r_{0}$ about $1 \AA$.

Of course the value of equilibrium distance is very strange if compared with the energy dissociation. But as reported in Ref. [20] this result is due to assumption of un-damped plasmon and the authors declare that "in a more realistic treatment the potential would tend more rapidly to zero". For this reason we believe that only the $D e$ value is reasonable.

Table 3 shows the new set of parameterization values correspondently to the alternative $D$ evaluation.

Fig. 3 shows the potential Eq. (27) evaluated assuming $D \mathrm{e}=50 \mathrm{eV}$.

We think that using this effective analytical potential we are able to explore a very large amount of situations that can occur within lattice simply varying the parameterization constant.

Now, let us discuss briefly about the role of parameters $D^{\prime}, r^{\prime}{ }_{0}$ and $A$.

Of course, $D^{\prime}$ is the parameter that controls the potential well, in fact for $D \mathrm{e}=9.34 \mathrm{eV}$ we put $D^{\prime}=0.28$,
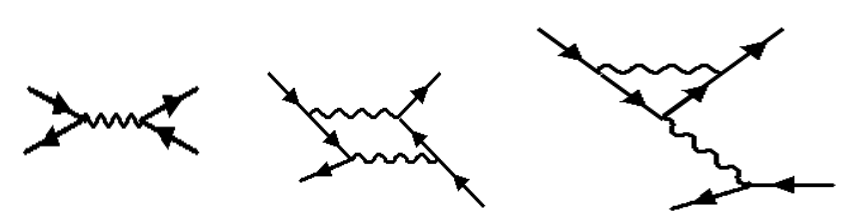

Fig. 3 Plasmon exchanges. Solid lines indicate deuterons and wiggly lines plasmons.

Table 3 The left column shows the physical quantities that must characterize the potential Eq. (27) where $D e$ has been evaluated in Ref. [20]; the right the model parameter values that are to be used in the expression (27) in order to obtain the physical values reported in the left column.

\begin{tabular}{ll}
\hline$\rho=0.165 \AA$ & $A=0.0001$ \\
$r_{0}=0.35 \AA$ & $r_{0}^{\prime}=0.99$ \\
$D e= \pm 50 \mathrm{eV}$ & $D^{\prime}=1.49$ \\
& $\gamma=1.04$ \\
\hline
\end{tabular}




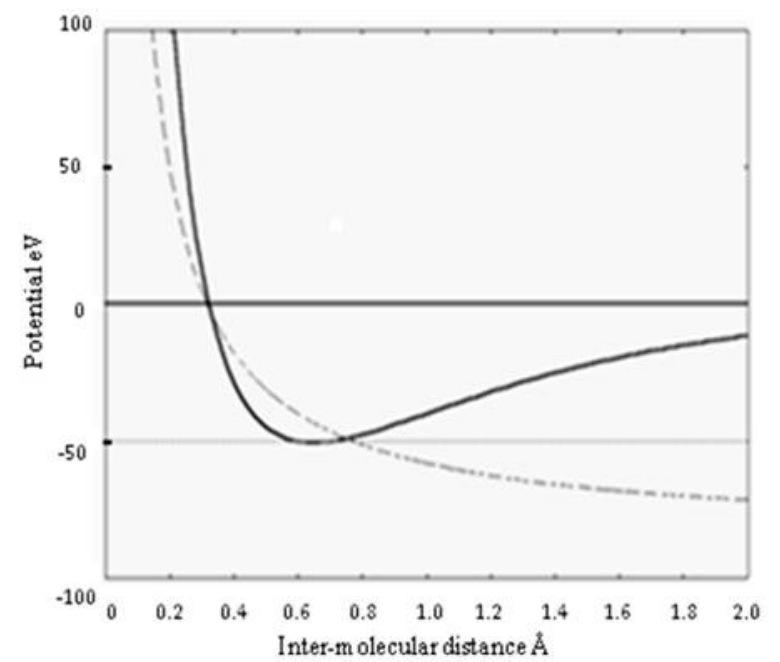

Fig. 4 The solid line shows the features of potential Eq. (27) computed using the values reported in Table 3 . The dashed line is the coulomb potential (26). In the $x$-axes, it is reported the distance in Bohr radius unit and on the $y$-axes the energy in $\mathrm{eV}$.

for $D e=50 \mathrm{eV}$ we have $D^{\prime}=1.49$. It means that this parameter depends mainly on screening efficiency.

Regarding $r^{\prime}{ }_{0}$ it is clear that it controls the zero crossing point of potential. In fact varying only this parameter the zero crossing point moves according to the increasing or decreasing of $r^{\prime}{ }_{0}$.

It is important to observe that $D^{\prime}$ and $r^{\prime}{ }_{0}$ are correlated. In fact it is reasonable to suppose that a bigger potential well corresponds to a smaller zero crossing point. Taking into account the value reported in Fig. 3, we can estimate this rough dependence:

$$
r_{0}^{\prime}=0.35^{*}\left(50 / D^{\prime}\right)
$$

Finally, regarding the parameters $A$, in the pictures shown previously it was put at 0.0001 . Moreover we can numerically prove that for small variation (of a factor less than 10) of this value, the potential does not change and its contribution appears relevant only at nuclear distance. Taking into account this observation, we can re-write this parameter as:

$$
A=H R
$$

where $R$ is about nuclear radius $\left(20 \mathrm{~F}=3,7610^{-4} a_{0}\right)$. It means that $H=0.265 \mathrm{eV}$.

This last value is about equal to the thermal energy of ions palladium that form the lattice Ref. [15]. For this reason we are conducted to put $H=K T$, where $K$ is the Boltzmann constant and $T$ is the lattice temperature.

\section{Results and Discussion}

A function having a Morse like trend seems to be the correct potential that is established between two deuterons within a lattice $[9,10,20]$. The expression (27) proposed in Section 4 is an analytical function that has the proper features of a Morse like potential linked with a Coulomb curve when the inter-nuclear distance approaches to zero. Using the potential Eq. (27) for the two different values of model parameters reported respectively in Tables 2 and 3, we have computed the $d$ - $d$ fusion rate (the nuclear reaction constant was $\left.10^{22} \sec ^{-1}\right)$. From data shown in Table 4, it is seen that the fusion rate appears in any case enough "great" or, in other words, "measurable". Moreover these values are in agreement with experimental data reported in Refs. [16, 17, 21]. More exactly in Refs. $[16,17]$ it has been reported a fusion rate of $10^{-23} \mathrm{sec}^{-1}$ for the reaction $D(d, p) T$, but no significant effects were observed in the neutron and gamma-ray measurements. While in Ref. [21] it has been reported a fusion rate of about $10^{-22} \mathrm{sec}^{-1}$ regarding the reaction $D(d, n) T$.

Of course, in order to better understand this phenomenon, it is necessary to distinguish the fusion rate expected by the theory according to the chain reaction, i.e. according to the reaction products expected. This issue has been (partially) clarified by Preparata [15].

Now let us briefly illustrate as the analytical expression of potential (27) can be extended to better handle the probably role of donor impurities on efficiency of screening.

Since the tunneling is enhanced by screening effect due to $d$-electron, it is clear that if we dope the lattice by means of donors atoms, the effect of electronic screening is increased. Considering by $J$ the impurities concentration, we have: 
Table 4 Fusion rate evaluated using the effective potential for different values of energy and for two different sets of model parameters.

\begin{tabular}{|c|c|c|c|}
\hline \multicolumn{2}{|c|}{$\begin{array}{l}A=0.0001 r^{\prime}{ }_{0}=0.99 \\
D^{\prime}=0.28 \gamma=1.04 \\
T(\mathrm{~K})=\text { Const } E(\mathrm{eV}) \\
\end{array}$} & \multicolumn{2}{|c|}{$\begin{array}{l}A=0.0001 r^{\prime}{ }_{0}=0.99 \\
D^{\prime}=1.49 \gamma=1.04 \\
T(\mathrm{~K})=\text { Const } E(\mathrm{eV})\end{array}$} \\
\hline$E \approx 50$ & $R \approx 6.32 \times 10^{-12}$ & $E \approx 50$ & $R \approx 3.02 \times 10^{-16}$ \\
\hline$E \approx 75$ & $R \approx 9.71 \times 10^{-18}$ & $E \approx 75$ & $R \approx 9.12 \times 10^{-17}$ \\
\hline$E \approx 100$ & $R \approx 9.95 \times 10^{-19}$ & $E \approx 100$ & $R \approx 8.2 \times 10^{-17}$ \\
\hline$E \approx 125$ & $R \approx 1.05 \times 10^{-20}$ & $E \approx 125$ & $R \approx 1.1 \times 10^{-18}$ \\
\hline$E \approx 150$ & $R \approx 5.6 \times 10^{-21}$ & $E \approx 150$ & $R \approx 9.15 \times 10^{-19}$ \\
\hline
\end{tabular}

$$
\mathrm{D}^{\prime} \rightarrow \mathrm{D}^{\prime}+\mathrm{GJ}
$$

where $G$ is a constant that depends on dopant-metal system.

The other parameter $r$, ${ }_{0}$ will change according to Eq. (31), while regarding A we suppose that in the presence of impurities we can write:

$$
A=J \xi K T
$$

where $\xi$ is a constant that depends on dopant-metal system.

From a point of engineering view, we can compute the new values of Eqs. (32) and (33) by measuring the bulk plasmon excitations in function of impurities density, and then following a set of theoretical calculations as reported in Ref. [20]. Finally using the potential (27) we will be able to evaluate the new fusion rate. The last point that will be evaluated in another work is the role of micro-crack. In fact, if within a lattice a micro-crack a local electrical field takes place that is able to increase the deuteron energy and then according to the values of Table 4 is able to enhance the fusion probability. To conclude, we can say that a cold fusion phenomenon is real. By means of standard theory of many bodies [20], it is theoretically possible to set aspect fusion rate of about $10^{-22} \mathrm{sec}^{-1}$, i.e. measurable values.

The very strange values of fusion rate as those reported by Refs. [2, 22] can be considered as errors, but if we take into account other mechanisms as micro-crack formation and/or the role of impurities, maybe, we will be able to understand these spikes and, maybe, reproduce them.

We have done a theoretical hypothesis of an oscillating double Coulomb barrier, so that the forbidden region could be avoided because it is not fixed.

At the moment, our aim is to find the right balance between the oscillations through time.

These topics will be further investigated in next works, where we will deal with the double Coulomb barrier for this reason the phenomenon of cold fusion is not constant.

\section{References}

[1] Paneth, F., and Peters, K. 1926. "Die Naturwissenschaften." Nature 118 (526).

[2] Fleischmann, M., and Pons. 1996. "Calibration of the Pd- $\mathrm{D}_{2} \mathrm{O}$ System: Protocol and Positive Feed-Back Effects." J. Chim. Phys. 93: 711.

[3] Iwamura, et al. 2002. "Elemental Analysis of Pd Complexes: Effects of $\mathrm{D}_{2}$ Gas Permeation." Jpn. J. Appl. Phys. A 41: 4642.

[4] Reifenschweiler, O. 1994. "Reduced Radioactivity of Tritium in Small Titanium Particles." Phys. Lett. A 184: 149.

[5] Reifenschweiler, O. 1996. "Some Experiments on the Decrease of Tritium Radioactivity." Fusion Technol. 30: 261.

[6] Rothwell, J. 1997. "Introduction to the Cold Fusion Experiments of Dr. Melvin Miles." Infinite Energy 3 (15/16): 27.

[7] Gamow, G. 1928. "Zeitschrift fur Physik" 51: 204.

[8] Sze, S. M., and Chang, C. Y. 1996. "Physics of Semiconductor Devices." In ULSI Technology, McGraw-Hill.

[9] Van Siclen, C. D. W., and Jones, S. E. 1986. "Piezonuclear Fusion in Isotopic Hydrogen Molecules." J. Phys. G: Nucl. Part. Phys. 12: 213.

[10] Jackson, J. D. 1957. "Catalysis of Nuclear Reactions between Hydrogen Isotopes by $\mu^{-}$Mesons." Phys. Rev. 106: 330-9. 
[11] Landau, and Lifshitz. 1965. Quantum Mechanics, Oxford Pergamon.

[12] Langer, R. E. 1937. "Collisional Transfer of Triplet Excitations between Helium Atoms." Phys. Rev. 69: 1301-9.

[13] Fleishmann, M., et al. 1989. "Electro-Chemically Induced Nuclear Fusion of Deuterium." Journal Electroanal. Chem. 261: 301-8.

[14] Horowitz, C. J. 1989. "Cold Fusion in Metallic Hydrogen and Normal Metals." Phys. Rev. C 40 (4): 1555-8.

[15] Tanzella, F. L. 1988. "Materials Issues of Loading Deuterium into Palladium and the Association with Excess Heat Production." In The Seventh International Conference on Cold Fusion, Vancouver, Canada: ENECO, Inc., Salt Lake City, UT.

[16] Johnson, K. H., and Clougherty, D. P. 1989. "Hydrogen-Hydrogen/Deuterium-Deuterium bonding in Palladium and the Superconducting/Electrochemical
Properties of PdH/PdD." Mod. Phys. Lett. B 3: 795.

[17] Preparata, G. 1995. QED Coherence in Matter, World Scientific.

[18] Frisone, F. 2001. "Theoretical Model of The Probability of Fusion between Deuterons within Deformed Crystalline Lattices with Micro-cracks at Room Temperature.” Fusion Technology 40 (2): 139-46.

[19] Christensen, O. B. 1989. "H-H Interactions in Pd." Phys. Rev. B 40: 1993-6.

[20] Baldo, M., and Pucci, R. 1989. "Plasmon Enhance Fusion." In Editrice Compositori, SIF, Conference Proceedings, Vol. 24, edited by R. A. Ricci, E. Sindoni and F. De Marco, Bologna, p. 283.

[21] Dragić, A., Marić, Z., and Vigier, J. P. 2000. "New Quantum Mechanical Tight Bound States and 'Cold Fusion' Experiments.” Phys. Lett. A 265: 163-7.

[22] Jones, S. E., et al. 1989. "Observation of Cold Nuclear Fusion in Condensed Matter." Nature 338: 737-40. 